\title{
Diagnostic Accuracy of Endoscopic Ultrasound-Guided Fine Needle Aspiration Cytology of Pancreatic Lesions
}

\author{
Hae Woon Baek · Min Jee Park \\ Ye-Young Rhee · Kyoung Bun Lee \\ Min A Kim • In Ae Park \\ Department of Pathology, Seoul National \\ University College of Medicine, Seoul, Korea
}

\author{
Received: September 5, 2014 \\ Revised: October 24, 2014 \\ Accepted: October 24, 2014 \\ Corresponding Author \\ In Ae Park, M.D. \\ Department of Pathology, Seoul National University \\ College of Medicine, 103 Daehak-ro, Jongno-gu, \\ Seoul 110-799, Korea \\ Tel: +82-2-2072-3312 \\ Fax: +82-2-743-5530 \\ E-mail: iapark@snu.ac.kr
}

\begin{abstract}
Background: Endoscopic ultrasound-guided fine needle aspiration cytology (EUS-FNAC) is currently the most commonly used procedure for obtaining cytologic specimens of the pancreas. It is accurate, minimally invasive, safe and cost-effective. However, there is discrepancy between cytological and surgical diagnoses. This study was aimed at evaluating the diagnostic accuracy of EUS-FNAC of the pancreas. Methods: We performed a retrospective review of 191 cases of pancreatic lesions initially diagnosed by EUS-FNAC with subsequent histological diagnosis between 2010 and 2012 in the Department of Pathology, Seoul National University Hospital. Cytologic and surgical diagnoses were categorized into five groups: negative, benign, atypical, malignant, and insufficient for diagnosis. Subsequently, 167 cases with satisfactory yield in both surgical and cytology specimens were statistically analyzed to determine correlations with diagnosis. Results: In comparison to surgical diagnoses, cytologic diagnoses were true-positive in 103 cases (61.7\%), true-negative in 28 cases (16.8\%), false-positive in 9 cases (5.4\%), and false-negative in 27 cases (16.1\%). The diagnostic accuracy was $78.4 \%$, sensitivity was $79.2 \%$, and specificity was $75.7 \%$. The positive predictive value was $92.0 \%$, and negative predictive value was $50.9 \%$. Conclusions: EUS-FNAC has high accuracy, sensitivity, specificity and positive predictive value. Overcoming the limitations of EUS-FNAC will make it a useful and reliable diagnostic tool for accurate evaluation of pancreatic lesions.
\end{abstract}

Key Words: Pancreas; Endoscopic ultrasound-guided fine needle aspiration cytology; Accuracy; Diagnosis
Pancreatic cancer is notorious for its poor prognosis, with a low overall 5-year survival rate of merely $8.7 \%$. It is the fifth leading cause of cancer-related mortality in South Korea ${ }^{1}$ because of the delayed detection of tumors, typical presentation at an advanced stage, and its aggressive disease behavior. Only 20\% of tumors are surgically resectable when detected. Remaining $80 \%$ of patients cannot help undergoing palliative therapy, the only treatment practical for those with unresectable tumor. In addition, pancreatic cancer has a poor response to chemotherapy and radiation therapy, increasing the complexity of patient management. Therefore, early detection is key in order to increase the survival rate of pancreatic cancer patients and to improve overall patient care.

Early detection, though it is crucial, is challenging since pancreatic cancer is usually asymptomatic in the initial stage and anatomically less accessible due to surrounding organs in the retroperitoneum. To overcome these limitations, imaging modalities such as abdominal ultrasound, computed tomography, magnetic resonance imaging, endoscopic retrograde cholangiopancreatography, endoscopic ultrasonography (EUS), and positron emission tomography have been used to localize the lesions.

For the detection of pancreato-biliary diseases, EUS is currently widely accepted. This technique enables precise visualization of the lesion and the ability to proficiently determine the depth of gastrointestinal malignancies. ${ }^{2}$ By combining the advantages of EUS with fine-needle aspiration cytology (FNAC) for the retrieval of specimens for pathologic diagnosis, EUS-FNAC has improved diagnostic capabilities. With EUS-FNAC, distinguishing pancreatic cancer from chronic pancreatitis, detection of tumor smaller than $2 \mathrm{~cm}$ and staging of the cancer are superior to those with the other modalities. EUS-FNAC has become the most popular technique with which to obtain cytology specimens and diagnose patients suspected to have pancreatic malignancy.

EUS-FNAC has been shown to be diagnostically useful, obviating unwarranted procedures and reducing costs. ${ }^{3}$ It is also 
minimally invasive and comparatively safe. The conclusion from a recently published meta-analysis stated that EUS-FNAC should be included in algorithms for the management of patients with solid pancreatic tumor due to its high accuracy as a diagnostic test. ${ }^{4}$

Although EUS-FNAC for diagnosis of solid pancreatic masses is recognized as 'a nearly perfect procedure, ${ }^{, 5}$ there are still several flaws that need to be ameliorated. Problematic issues may arise due to limited skills of the endoscopy operator in terms of insufficient yield and targeting-error, misinterpretation and misdiagnosis by pathologists and absence of on-site cytopathologists for adequacy assessment.

This study was aimed to evaluate the diagnostic accuracy of EUS-FNAC of the pancreas and to further investigate the reason for incorrect diagnosis by comparison with confirmed histological diagnosis. The reason which we focused on the discrepancy between cytological and surgical specimen diagnosis was to identify the pitfalls that pathologists may face during diagnosis. In some patients, the initial diagnostic cytology specimen may be the only material that has viable tumor cells for diagnosis if the tumor is unresectable or they receive neoadjuvant therapy prior to resection. This situation emphasizes the importance of the accuracy of cytopathologic diagnosis. Furthermore, on-site cytopathologic assessment for adequacy is usually not performed in South Korea for financial reasons, ${ }^{6}$ making it even more challenging for pathologists in Korea to diagnose cytological specimens.

Retrospective examination was performed in this study, correlating cytology-histology diagnosis of pancreatic lesions obtained by EUS-FNAC with several clinicopathologic variables. In doing so, we hope to optimize the accuracy of cytological diagnosis in an effort to improve the care of pancreatic cancer patients.

\section{MATERIALS AND METHODS}

A retrospective study was carried out to review 191 cases of EUS-FNAC of pancreatic lesions between January 2010 and December 2012 in Seoul National University Hospital. This study was approved by the Seoul National University Hospital Institutional Review Board (IRB Study No. H-1408-022-601).

\section{Case selection}

The data from 579 patients who underwent pancreatic FNAC over a 36-month period (January 2010 to December 2012) were obtained by computerized search of PathPACS (Humintec, Suwon, Korea), a database used at the Department of Pathology of Seoul National University Hospital. Cases with no follow-up biopsy or surgical resection were excluded from this study, leaving 207 cases. Among those cases, 191 patients underwent EUS-FNAC. Specimens from 8 patients were retrieved by intraoperative brush cytology, 4 from ultrasoundguided gun biopsy, and 4 were unclear with no specific record of the procedure. In this study, only patients who underwent EUSFNAC were included. For statistical analysis, patients surgically diagnosed as 'atypical' or 'insufficient for diagnosis (IFD)' were further excluded since those diagnoses were not as satisfactory as confirmation of cytologic diagnosis. The remaining 167 cases were analyzed to assess the diagnostic accuracy of EUS-FNAC.

\section{Procedure: EUS-guided fine-needle aspiration and specimen preparation}

Radial and linear endoscopic ultrasonographies were used for EUS-FNAC. Fine-needle aspiration was performed by gastroenterologists of Seoul National University Hospital. For cytopathologic analysis, the aspirated specimen was smeared onto glass slides and fixed in 95\% ethanol, followed by Papanicolaou staining and Diff-Quick staining. A cell block was prepared in 2 cases using a standard protocol.

\section{Cytologic diagnosis}

Diagnoses were made by several pathologists at Seoul National University Hospital. The specimen was initially rated either as adequate or inadequate. Suboptimal specimens with less than minimal pancreatic tissue needed for diagnosis were rated inadequate. Adequate samples were then categorized into four groups: negative for malignancy, benign lesion, atypical, and malignant neoplasm. Altogether there were five groups, including inadequate samples grouped as 'IFD.' Diagnosis of EUSFNAC was then compared with subsequent corresponding histologic diagnosis.

\section{Retrospective review of cases with discrepancy}

Of the 167 included cases, 36 showed major discrepancy between cytological diagnosis and surgical diagnosis. Retrospective review of those slides was conducted by three pathologists: the corresponding author (I.A.P.) and K.B.L, who are experienced pathologists, and the first author (H.W.B.). For non-biased review results, final histological diagnosis was blinded.

\section{Statistical analysis}

The data on patient sex, age, type of procedure, diagnosis for cytology and surgical specimen, and site of aspiration were ana- 
lyzed using Microsoft Excel 2007 calculation sheets. For patients with several cytological specimens obtained from the same site on the same day, that with the most corresponding result with final surgical diagnosis was included in the data analysis. The diagnostic accuracy, sensitivity, specificity, positive and negative predictive values, false-positive rate, false-negative rate, and false-discovery rate of EUS-FNAC results were calculated. For the statistical analysis, cytology and surgical diagnosis of 'benign lesion' were categorized as a negative result, meaning 'negative for tumor,' while 'atypical' and 'suspicious for malignancy' (the gray-zone) were considered and categorized as 'positive for tumor.' Although surgical diagnoses of 'atypical' and 'IFD' were excluded from statistical analysis, we included 'atypical' and 'IFD' diagnoses of cytology specimens for broader evaluation.

\section{RESULTS}

\section{Patient characteristics}

Among 191 patients who underwent EUS-FNAC, the male to female ratio was 0.95 , with 93 males and 98 females. The median age of the patients was 60.25 years, ranging from 20 to

Table 1. Correlation of EUS-FNAC diagnosis and corresponding final histological diagnosis

\begin{tabular}{|c|c|c|c|c|}
\hline Cytology diagnosis & \multicolumn{2}{|c|}{ Surgical diagnosis } & \multirow{2}{*}{$\frac{\text { No. of cases }}{15}$} & \multirow{2}{*}{$\begin{array}{c}\text { Category } \\
\text { True-negative }\end{array}$} \\
\hline Negative & Non-neoplastic lesion & & & \\
\hline \multirow[t]{5}{*}{ (35 cases, 18.3\%) } & Benign lesion & & 2 & True-negative \\
\hline & Atypical (undetermined) & & 2 & Excluded \\
\hline & Malignant neoplasm $(n=15)$ & Ductal adenocarcinoma & 13 & False-negative \\
\hline & & Neuroendocrine tumor & 1 & \\
\hline & & IPMN & 1 & \\
\hline \multirow[t]{4}{*}{ (5 cases, $2.6 \%)$} & Non-neoplastic lesion & & 0 & True-negative \\
\hline & Benign lesion & & 5 & True-negative \\
\hline & Atypical (undetermined) & & 0 & Excluded \\
\hline & Malignant neoplasm & & 0 & False-negative \\
\hline Atypical (undetermined) & TIFD & & 0 & Excluded \\
\hline \multirow[t]{6}{*}{ (37 cases, 19.4\%) } & Non-neoplastic lesion & & 4 & False-positive \\
\hline & Benign lesion & & 1 & False-positive \\
\hline & Malignant neoplasm $(n=24)$ & Ductal adenocarcinoma & 18 & True positive \\
\hline & & Neuroendocrine tumor & 2 & \\
\hline & & IPMN & 2 & \\
\hline & & Malignant tumor, unspecified & 2 & \\
\hline Malignant neoplasm & TIFD & & 3 & Excluded \\
\hline \multirow[t]{9}{*}{ (94 cases, 49.2\%) } & Non-neoplastic lesion & & 4 & False-positive \\
\hline & Benign lesion & & 0 & False-positive \\
\hline & Atypical (undetermined) & & 9 & Excluded \\
\hline & Malignant neoplasm $(n=79)$ & Ductal adenocarcinoma & 57 & True-positive \\
\hline & & Neuroendocrine tumor & 10 & \\
\hline & & Carcinoma & 4 & \\
\hline & & IPMN & 3 & \\
\hline & & Solid-pseudopapillary neoplasm & 2 & \\
\hline & & Metastatic leiomyosarcoma & 1 & \\
\hline Insufficient for diagnosis & TIFD & & 2 & Excluded \\
\hline \multirow[t]{7}{*}{ (20 cases, $10.5 \%)$} & Non-neoplastic lesion & & 5 & True-negative \\
\hline & Benign lesion & & 1 & True-negative \\
\hline & Atypical (undetermined) & & 1 & Excluded \\
\hline & Malignant neoplasm $(n=12)$ & Ductal adenocarcinoma & 10 & False-negative \\
\hline & & IPMN & 1 & \\
\hline & & Malignant tumor, unspecified & 1 & \\
\hline & TIFD & & 1 & Excluded \\
\hline Total & & & 191 & \\
\hline
\end{tabular}

TIFD, tissue insufficient for diagnosis; IPMN, intraductal papillary mucinous neoplasm. 
82 years.

Cytologic results consisted of 35 cases $(18.3 \%)$ of 'negative for tumor,' 5 cases (2.6\%) of 'benign lesion,' 37 cases (19.4\%) of 'atypical,' 94 cases (49.2\%) of 'malignant neoplasm,' and 20 cases $(10.5 \%)$ of 'IFD' (Table 1). Specific diagnoses of cytologically 'malignant neoplasm' included 53 cases of ductal adenocarcinoma, 28 cases of 'malignant tumor, unspecified,' 10 cases of neuroendocrine neoplasm, 2 cases of mucinous neoplasm, and 1 case of squamous cell carcinoma (Table 2).

Histological results consisted of 28 cases $(14.7 \%)$ of 'negative for tumor,' 9 cases (4.7\%) of 'benign lesion,' 17 cases $(8.9 \%)$ of 'atypical,' 130 cases $(68.1 \%)$ of 'malignant neoplasm,' and 7 cases $(3.6 \%)$ of 'tissue insufficient for diagnosis (TIFD)' (Table 1).

\section{Comparison between cytological and surgical diagnoses}

In a comparison of cytology-surgical diagnoses (Table 1), 35 cases of negative cytology diagnosis were surgically diagnosed as 'non-neoplastic lesion' in 15 cases and 'benign lesion' in 2 cases. Those cases were classified as true-negative results. On the contrary, there were 15 false-negative cases showing major discrepancy, which were initially diagnosed negative on cytology but malignant on surgical specimen. Two cases with surgical diagnosis of 'atypical (undetermined)' and 1 case of 'TIFD' were excluded from the statistical analysis due to unsatisfactory results of confirmation. Five cases were cytologically diagnosed as 'benign lesion' and were also diagnosed as 'benign lesion' on surgical diagnosis. These cases were classified as true-negative.

There were 37 cytologically 'atypical (undetermined)' cases. Surgical diagnosis of these cases was true-positive ('malignant neoplasm') in 24 cases and false-positive in 5 cases ( 4 'benign lesion' and one 'non-neoplastic lesion'). Eight cases that were surgically diagnosed 'TIFD' ( 3 cases) and 'atypical' (5 cases) were likewise excluded. As for cytological specimens diagnosed 'malignant neoplasm', 79 of 94 cases were true-positive with surgical diagnosis of 'malignant neoplasm.' There were 4 false-positive results for specimens surgically diagnosed as 'non-neoplastic lesion.' In the same manner as the other categories, 9 cases of surgically 'atypical' and 2 cases of surgically 'TIFD' were excluded.

Table 2. Specific diagnoses of cytologically 'malignant neoplasm'

\begin{tabular}{lc}
\hline Cytology diagnosis & Total \\
\hline Ductal adenocarcinoma & 53 \\
Malignant tumor, unspecified & 28 \\
Neuroendocrine neoplasm & 10 \\
Mucinous neoplasm & 2 \\
Squamous cell carcinoma & 1 \\
Total & 94 \\
\hline
\end{tabular}

Among 20 cases of cytologically 'IFD' cases, 6 were truenegative (five surgically 'non-neoplastic lesion' and one 'benign lesion'), while 12 cases were false-negative with a surgical diagnosis of 'malignant neoplasm.' Cases with surgical diagnosis of 'IFD' (1 case) and 'atypical' (1 case) were excluded. Last of all, there was a case that was cytologically diagnosed as 'suspicious for neuroendocrine tumor' but surgically diagnosed as solid pseudopapillary neoplasm, which are distinctive in diagnosis. Despite the discrepancy, this case was classified as true-positive in order to acknowledge the cytological diagnosis for recognizing a malignancy.

\section{Statistical results}

According to the data, the 167 cases remaining after applying exclusion criteria were true-positive in 103 cases (61.7\%), truenegative in 28 cases (16.8\%), false-positive in 9 cases $(5.4 \%)$, and false-negative in 27 cases $(16.1 \%)$. The diagnostic accuracy was $78.4 \%$, sensitivity was $79.2 \%$, and specificity was $75.7 \%$. The positive predictive value was $92.0 \%$, and negative predictive value was $50.9 \%$. The false-positive rate was $24.3 \%$, false-negative rate was $11.6 \%$, and false discovery rate was $8.0 \%$ (Table 3 ).

\section{Analysis of discrepant cases: false-positives and false- negatives}

Thirty-five cases showed discrepancy in cytological-surgical correlation, and 9 cases among them were false-positive (Table 4). Cytologically diagnosed 'atypical' specimens were surgically diagnosed negative in 2 cases and lymphoplasmacytic sclerosing pancreatitis in 2 cases. Two cytologic diagnoses of 'suspected carcinoma,' one 'carcinoma,' and one 'adenocarcinoma' were also false-positive, later surgically diagnosed as 'negative for tumor.'

Table 3. Statistical analysis of 167 cases $^{a}$

\begin{tabular}{lcl}
\hline Category & Percentage & \multicolumn{1}{c}{ Equation } \\
\hline TP (103 cases) & 61.7 & $\mathrm{~N} / \mathrm{A}$ \\
TN (28 cases) & 16.8 & $\mathrm{~N} / \mathrm{A}$ \\
FP (9 cases) & 5.4 & $\mathrm{~N} / \mathrm{A}$ \\
FN (27 cases) & 16.1 & $\mathrm{~N} / \mathrm{A}$ \\
Diagnostic accuracy & 78.4 & $(\mathrm{TP}+\mathrm{TN}) /(\mathrm{TP}+\mathrm{TN}+\mathrm{FP}+\mathrm{FN}) \times 100$ \\
Sensitivity & 79.2 & $\mathrm{TP} /(\mathrm{TP}+\mathrm{FN}) \times 100$ \\
Specificity & 75.7 & $\mathrm{TN} /(\mathrm{TN}+\mathrm{FP}) \times 100$ \\
Positive predictive value & 92.0 & $\mathrm{TP} /(\mathrm{TP}+\mathrm{FP})$ \\
Negative predictive value & 50.9 & $\mathrm{TN} /(\mathrm{TN}+\mathrm{FN})$ \\
FP rate & 24.3 & $\mathrm{FP} /(\mathrm{FP}+\mathrm{TN})$ \\
FN rate & 11.6 & $\mathrm{FN} /(\mathrm{TP}+\mathrm{FN})$ \\
\hline
\end{tabular}

TP, true positive; TN, true negative; FP, false positive; FN, false negative; N/ A, not applicable.

a'Surgically 'atypical' (17 cases) and 'tissue insufficient for diagnosis' (7 cases) cases are excluded. 
Table 4. Discrepant cases with a false-positive or false-negative cytology diagnosis

\begin{tabular}{|c|c|c|c|c|c|c|c|}
\hline \multicolumn{2}{|c|}{ Category $^{a}$} & \multirow{2}{*}{$\frac{\text { Case No. }}{1}$} & \multirow{2}{*}{$\frac{\text { Sex }}{F}$} & \multirow{2}{*}{$\frac{\text { Age }(y r)}{70}$} & \multirow{2}{*}{$\begin{array}{c}\text { Location } \\
\text { Neck }\end{array}$} & \multirow{2}{*}{$\frac{\text { Cytology diagnosis }}{\text { IFD }}$} & \multirow{2}{*}{$\begin{array}{c}\text { Surgical diagnosis } \\
\text { Ductal adenocarcinoma }\end{array}$} \\
\hline 1 & False-negative & & & & & & \\
\hline & & 2 & $\mathrm{~F}$ & 53 & Uncinate & IFD & Ductal adenocarcinoma \\
\hline & & 3 & M & 69 & Tail & IFD & Ductal adenocarcinoma \\
\hline & & 4 & $\mathrm{~F}$ & 65 & Body & IFD & Ductal adenocarcinoma \\
\hline & & 5 & M & 76 & Body & IFD & Ductal adenocarcinoma \\
\hline & & 6 & $M$ & 58 & Head & IFD & Ductal adenocarcinoma \\
\hline & & 7 & M & 77 & Body & IFD & Ductal adenocarcinoma \\
\hline & & 8 & M & 76 & Body & IFD & Ductal adenocarcinoma \\
\hline & & 9 & $\mathrm{~F}$ & 69 & Neck & IFD & Ductal adenocarcinoma \\
\hline & & 10 & M & 71 & Body & IFD & IPMN \\
\hline & & 11 & $\mathrm{~F}$ & 70 & Main p-duct & IFD & Malignancy, unspecified \\
\hline & & 12 & $\mathrm{~F}$ & 50 & Body & IFD & Neuroendocrine tumor \\
\hline \multirow[t]{16}{*}{2} & False-positive & 13 & M & 75 & Body & Suspected carcinoma & Negative for tumor \\
\hline & & 14 & M & 75 & Body & Suspected carcinoma & Negative for tumor \\
\hline & & 15 & M & 60 & Neck & Carcinoma & Negative for tumor \\
\hline & & 16 & $\mathrm{~F}$ & 58 & Body & Adenocarcinoma & Negative for tumor \\
\hline & & 17 & $\mathrm{~F}$ & 82 & Head & Atypical & Negative for tumor \\
\hline & & 18 & $\mathrm{~F}$ & 38 & Body & Atypical & Negative for tumor \\
\hline & False-negative & 19 & M & 57 & Tail & Negative for tumor & Ductal adenocarcinoma \\
\hline & & 20 & $\mathrm{~F}$ & 61 & Uncinate & Negative for tumor & Ductal adenocarcinoma \\
\hline & & 21 & $\mathrm{~F}$ & 51 & Tail & Negative for tumor & Ductal adenocarcinoma \\
\hline & & 22 & $\mathrm{~F}$ & 41 & Head & Negative for tumor & Ductal adenocarcinoma \\
\hline & & 23 & M & 77 & Body & Negative for tumor & Ductal adenocarcinoma \\
\hline & & 24 & $\mathrm{~F}$ & 67 & Head & Negative for tumor & Ductal adenocarcinoma \\
\hline & & 25 & $\mathrm{~F}$ & 72 & Head & Negative for tumor & Ductal adenocarcinoma \\
\hline & & 26 & M & 57 & Tail & Negative for tumor & Ductal adenocarcinoma \\
\hline & & 27 & $\mathrm{~F}$ & 73 & Uncinate & Negative for tumor & Ductal adenocarcinoma \\
\hline & & 28 & M & 64 & Body & Negative for tumor & Ductal adenocarcinoma \\
\hline \multirow[t]{7}{*}{3} & False-positive & 29 & M & 50 & Body & Atypical & Lymphoplasmocytic sclerosing pancreatitis \\
\hline & & 30 & $\mathrm{~F}$ & 60 & Distal part & Atypical & Lymphoplasmocytic sclerosing pancreatitis \\
\hline & False-negative & 31 & M & 76 & Body & Negative for tumor & Ductal adenocarcinoma \\
\hline & & 32 & M & 56 & Head & Negative for tumor & Ductal adenocarcinoma \\
\hline & & 33 & M & 61 & Tail & Negative for tumor & Ductal adenocarcinoma \\
\hline & & 34 & $\mathrm{~F}$ & 58 & Tail & Negative for tumor & Ductal adenocarcinoma \\
\hline & & 35 & $\mathrm{~F}$ & 70 & Uncinate & Negative for tumor & IPMN \\
\hline
\end{tabular}

F, female; IFD, insufficient for diagnosis; M, male; IPMN, intraductal papillary mucinous neoplasm.

aDiscrepancy category: 1) Insufficient for diagnosis (cytology specimen of too few cells), 2) Technical targeting error (normal parenchyma or other non-lesion area aspirated), and 3) Misdiagnosis by pathologists.

There were 27 cases that resulted in false-negative results (Table 4). Cytologically diagnosed 'negative for tumor' or 'IFD' were surgically diagnosed ductal adenocarcinoma in 23 cases, intraductal papillary mucinous neoplasm in 2 cases, neuroendocrine tumor in 1 case, and 'malignancy, unspecified' in 1 case.

We reviewed the cytology slides for discrepant cases in order to analyze reasons for such results and assigned cases to the following three main categories: 1) IFD (cytologic specimen with too few cells), 2) technical targeting error (aspiration of normal parenchyma or other non-lesion area), and 3) misdiagnosis by pathologists. The number of cases that fall into these categories is $12(7.2 \%), 16(9.6 \%)$, and 7 (4.2\%), respectively (Table 4).

Through a group review by 3 pathologists, the reasons for the discrepancy in each case in category 3 were analyzed. In 3 cases that were histologically adenocarcinoma but cytologically diagnosed negative, obvious malignant cell clusters that resembled adenocarcinoma were observed (cases Nos. 31-34) (Table 4, Fig. 1A). These cases were analyzed as misdiagnosis due to omission of tumor cells by inattentive screening. Another case diagnosed 'histologically adenocarcinoma but cytologically negative' (case No. 32) (Table 4, Fig. 1B, C) showed some malignant cell clusters that were intermixed with and camouflaged by a massive amount of benign parenchymal cells. This case was analyzed as a misdiagnosis due to misinterpretation of the pathologist.

Intraductal papillary mucinous neoplasm was diagnosed as negative in one case (case No. 35) (Table 4, Fig. 1D). Some mucin-producing epithelial cells with suspicious atypism were observed from the slide review, leading us to also analyze this case 

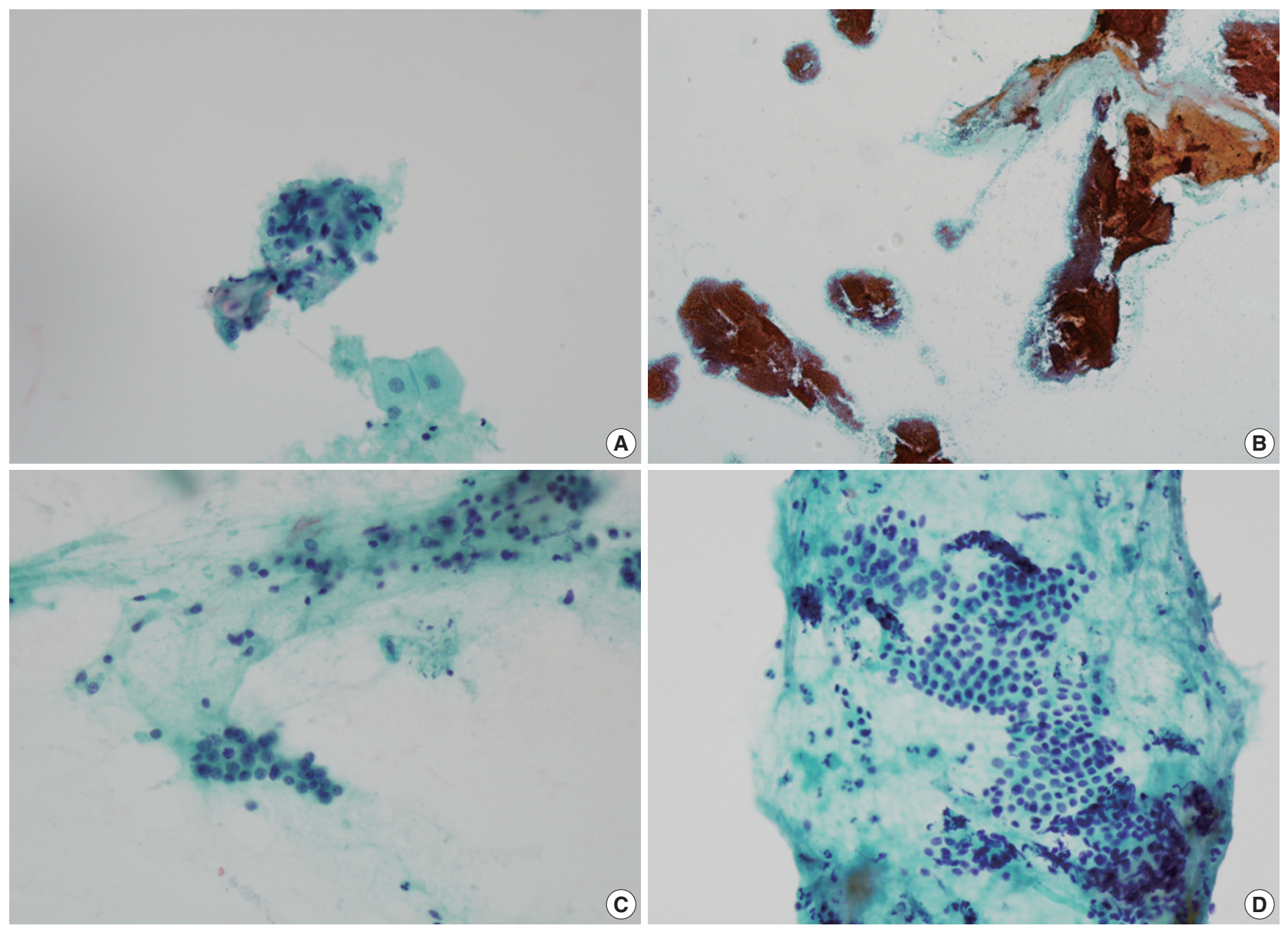

Fig. 1. Cytologic specimens with false-negative discrepant results (Table 4). (A) Case No. 31 with histological diagnosis of ductal adenocarinoma. Obvious malignant cell clusters that resemble adenocarcinoma. (B, C) Case No. 32 with histological diagnosis of ductal adenocarinoma. Malignant cell clusters are intermixed with and camouflaged by a massive amount of benign parenchymal cells. (D) Case No. 35 with histological diagnosis of intraductal papillary mucinous neoplasm. Some mucin-producing epithelial cells with suspicious atypism are observed.

as a misinterpretation by the pathologist.

Three cases were surgically diagnosed as 'consistent with lymphoplasmacytic sclerosing pancreatitis.' Among them, 2 cases were cytologically misdiagnosed as 'atypical,' resulting in discrepancy (cases Nos. 29 and 30) (Table 4). The remaining case was cytologically diagnosed 'negative for tumor,' which was categorized as a true-negative result in this study. In cytology specimens, inflammatory infiltrate consisted mainly of lymphocytes and plasma cells were observed.

A cytology specimen that was diagnosed as 'a few atypical cells' and histologically diagnosed schwannoma (Fig. 2) was also reviewed. This case was not included in category 3 since it is not a serious misdiagnosis. Cytopathologic features presented mostly in tissue fragments or in fascicles, with cells fusiform and an elongated shape with poorly defined cell borders. Cytology showed low nuclear-cytoplasmic ratio with long and wavy nuclei. Nucleoli were inconspicuous, and cytoplasm was pale.

The anatomical site of aspiration in category 1 was the body in 6 cases, neck in 2 cases, uncinate process in 1 case, head in 1 case, tail in 1 case, and 'main p-duct' in 1 case. For category 2, aspirations were conducted in the body in 5 cases, neck in 4 cases, tail in 3 cases, uncinate process in 2 cases, and neck in 1 case. Category 3 cases were collected from the body in 3 cases, tail in 2 cases, neck in 1 case, uncinate process in 1 case, and distal part in 1 case (Table 4).

\section{DISCUSSION}

EUS-FNAC for pancreatic solid tumor is widely performed and has been shown to be useful. ${ }^{3,5,7,8}$ As EUS-FNAC has gained acknowledgement as the gold standard for obtaining patient specimens, the importance and demand for optimization of EUS- 


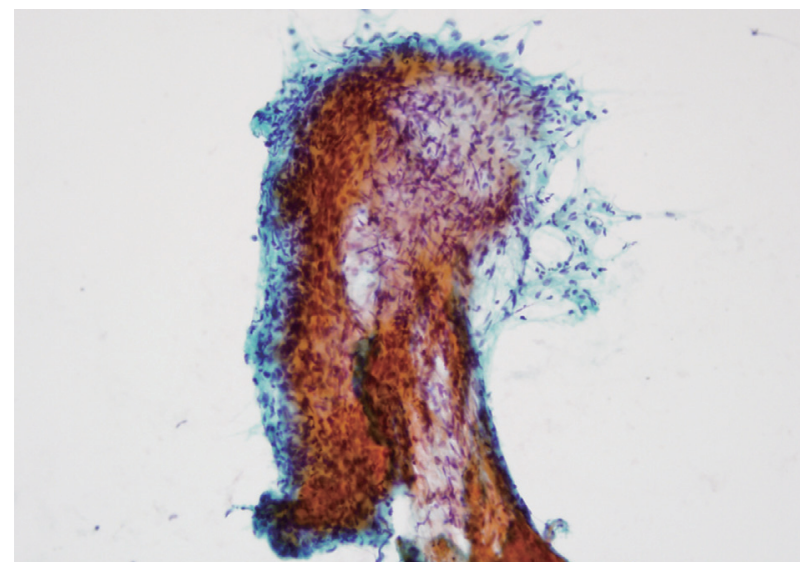

Fig. 2. Cytologic specimen with corresponding histological diagnosis of schwannoma. Cytopathologic features present mostly in tissue fragments or in fascicles, with cells fusiform and elongated with poorly defined cell borders, a low nuclear-cytoplasmic ratio with long and wavy nuclei, inconspicuous nucleoli and pale cytoplasm.

FNAC increase. We, given the relatively large number of cases of pancreatic FNAC in South Korea, have recognized that follow up and review are needed of past EUS-FNAC results in order to determine the validity of the examination process. The aim of the current study was to contribute to the advancement of management for pancreatic cancer patients by improving the detection and diagnosis results.

To analyze the accuracy, we investigated the diagnosis of EUS-FNAC by comparing with final diagnoses confirmed by histological examination of biopsy or surgically resected specimens. During evaluation of the diagnoses made by EUSFNAC, we emphasized the agreement with the final diagnoses.

In our data, $61.7 \%$ of cases were true-positive and $16.8 \%$ of cases were true-negative, with false-negative and false-positive cases comprising only $21.6 \%$, which is acceptably low considering that most were due to adequacy problems with the EUSFNAC specimen. As a result, diagnostic accuracy, sensitivity, and specificity were $78.4 \%, 79.2 \%$, and $75.7 \%$ respectively. The positive predictive value was $92.0 \%$, and negative predictive value was $50.9 \%$. The false-positive rate was $24.3 \%$, falsenegative rate was $11.6 \%$, and false discovery rate was $8.0 \%$. According to Yoshinaga et al., 5 a medical literature review to evaluate the role of EUS-FNAC for diagnosis of solid pancreatic masses showed $78 \%-95 \%$ sensitivity, $75 \%-100 \%$ specificity, 98\%-100\% positive predictive value, $46 \%-80 \%$ negative predictive value, and $78 \%-95 \%$ accuracy. In comparison of this data with data from several other studies, ${ }^{9-12}$ our study showed lower but within the range values of diagnostic accuracy, sensi- tivity and specificity. The positive predictive value was $12 \%$ higher than the upper margin, but the negative predictive value was lower than the mean. Our overall results were affirmative and supportive of the continued use of EUS-FNAC for pancreatic lesion, but it is apparently lower than other institutes. This encourages us to look for an explanation and identify mechanisms for improvement. Such relatively poor result may be due to the way of manipulating raw data during patient selection and categorization of diagnoses. By adjusting our methods to be more identical to those of other studies, we may have gained more satisfying results, similar to those of other institutes.

After confirming that the overall results were comparatively favorable in our study, we focused on the cases that showed discrepancy in order to identify the pitfalls of diagnosis and further improve the cytologic diagnosis. We reviewed the slides and analyzed the diagnoses of 35 cases in which the cytologic diagnosis did not concur with the surgical diagnosis. Among those cases, 12 were due to insufficient aspiration of cells for diagnosis (category 1), and 16 were due to targeting error (category 2), containing only benign parenchyma instead of tumor. The remaining 7 discrepant cases (category 3) were due to misinterpretation and misdiagnosis by pathologists.

Categories 1 and 2 results indicate aspiration failures caused by technical variables, such as operator skills/experiences, tumor type and location. With regard to location, cases were aspirated most often from the body (11 cases), followed by head ( 5 cases) and tail ( 4 cases). The interpretation is that the aspirations from these regions have a tendency to be insufficient due to poor accessibility and technical difficulty. However, it could just be a matter of fraction, representing the most common location of the suspected tumor in this study population. The most common cytologic diagnosis for categories 1 and 2 was 'adenocarcinoma' (19 cases), followed by 'negative for tumor' (5 cases), neuroendocrine tumor (1 case), intraductal papillary neoplasm (1 case), and 1 case of 'malignancy, unspecified.' Considering that adenocarcinoma was the most common diagnosis overall (adequate/inadequate, discrepant/non-discrepant), tumor type may have less impact on aspiration failure. In addition, we took into consideration that cases of intraductal papillary mucinous neoplasm may have been underdiagnosed as 'benign lesion' in this study. Therefore, we concluded that the relativity of tumor type and diagnostic accuracy is still ambiguous. Also, we assumed that there are significant influences from variability in operator skills and tumor size on EUS-FNAC results. However, we could not analyze such data because the electric medical records did not document the specific operator's name and tumor 
size in all cases.

For category 3, the misdiagnosed cases, we reviewed the slides to identify the factor that led to such discrepancy. Of 7 cases, four were histologically adenocarcinoma but cytologically diagnosed negative. Meticulous observation led to identification of some obviously malignant cells that resemble adenocarcinoma in 3 cases (cases Nos. 31-34) (Table 4, Fig. 1A). In these cases, we think the misdiagnosis was due to screening failure and simple exclusion of the applicable areas on the slide. On the other hand, one histologically adenocarcinoma but cytologically diagnosed 'negative' case was actually challenging (case No. 32) (Table 4, Fig. 1B, C). As reviewers retrospectively observing the collection of 'discrepant' cases only, it was not difficult to interpret this case as malignant since we knew that something must be wrong. However, tumor cells in this case were intermixed with and camouflaged by a massive amount of benign parenchymal cells, making the malignancy ambiguous. In a situation like this, a pathologist may be discouraged and hesitant to conclude a diagnosis of definite cancer.

Intraductal papillary mucinous neoplasm was misdiagnosed as negative in 1 case (case No. 35) (Table 4, Fig. 1D). From review of the slide, we recognized some mucin-producing epithelial cells with suspicious atypism. We assumed that the discrepancy in this case was due to diagnosis by a relatively inexperienced pathologist, leading to misinterpretation.

There were 3 cases surgically diagnosed as 'consistent with lymphoplasmacytic sclerosing pancreatitis.' Among them, 2 cases were cytologically misdiagnosed as 'atypical,' resulting in discrepancy (cases Nos. 29 and 30) (Table 4). The remaining case was cytologically diagnosed 'negative for tumor' and was categorized as a true negative result. Lymphoplasmacytic sclerosing pancreatitis, a form of chronic pancreatitis with mixed inflammatory infiltrate, clinically mimics pancreatic cancer. Preoperative detection is important because lymphoplasmacytic sclerosing pancreatitis patients usually respond to steroid therapy with reversible improvement in pancreatic morphology and function. ${ }^{13}$ In our cases, inflammatory infiltrate consisting mainly of lymphocytes and plasma cells was observed. According to Abraham et al., ${ }^{14}$ this infiltrate may also contain some macrophages and occasionally neutrophilic and eosinophilic granulocytes. Although the role of FNAC is mainly to distinguish malignant from benign cells, it is worth considering the possibility of lymphoplasmacytic sclerosing pancreatitis when investigators recognize such microscopic features because patients will benefit from earlier initiation of therapy.

We also reviewed a case of schwannoma that was cytological- ly diagnosed as 'a few atypical cells' (Fig. 2). Cytopathologic features presented mostly in tissue fragments or in fascicles, with cells fusiform and elongated with poorly defined cell borders. The cells showed a low nuclear-cytoplasmic ratio with long and wavy nuclei. Nucleoli were inconspicuous, and cytoplasm was pale. Pancreatic schwannoma is an extremely rare neoplasm, with only 47 cases reported in the English literature in the last three decades. ${ }^{15}$ Therefore, it is not routine for pathologists to suspect such schwannoma when screening. However, the possibility that cells are mesenchymal should be considered, which may suggest the diagnosis.

Navina et al. ${ }^{16}$ and Kim et al. ${ }^{6}$ reported that absence of an immediate on-site cytopathologist is not critical, and they found no association with on-site evaluation and specimen cellularity. However, many groups, for example, Fisher et al. ${ }^{8}$, have reported that on-site evaluation was relatively accurate $(77.5 \%)$ and highly specific for malignancy $(100 \%)$, significantly contributing to the efficiency and accuracy of the procedures. With respect to our lower diagnostic accuracy in comparison to those of other institutes, absence of on-site evaluation may be the cause because 28 of 35 discrepant cases in our study were due to unsatisfactory specimens. We presume that we could have benefited from on-site evaluation to improve diagnostic results and reduce the number of discrepant cases. In our opinion, having immediate on-site evaluation would be beneficial and valuable, if circumstances allowed.

The design of this study was limited by the fact that it was a single-center retrospective review of a relatively small number of consecutive cases over a 36-month period. Thorough adequacy assessment of pancreatic EUS-FNAC was impossible since only one representative specimen of patients with multiple aspirations was analyzed. Had we not excluded numerous 'IFD' cases, there would have been a greater amount of useful data, which would have reduced the impact of incorrect results caused by technical difficulty. Also, data identifying the operator and pathologist should have been retrieved to analyze artificial error that depends on skill and experience. Further study on cases with discrepancy aimed to identify the pitfalls of diagnosis should involve more cases of misdiagnosis, increasing the power of the analysis. Awareness of such pitfalls is important because it increases diagnostic confidence, resulting in improved accuracy.

In summary, the diagnostic accuracy of EUS-FNAC for obtaining pancreatic specimens suspicious of malignancy was confirmed to be high in this study. Diagnostic accuracy, sensitivity, and specificity were $78.4 \%, 79.2 \%$, and $75.7 \%$ respectively. Although 35 of 191 cases showed discrepancy in cytology-his- 
tology diagnosis, most were due to insufficient aspiration or mistargeted aspiration of cells, both of which preclude proper examination. Therefore, we conclude that EUS-FNAC is reliable and accurate. Based on these results, pathologists can be assured of their diagnosis, as EUS-FNAC provides a desirable representation of the specimen. However, particular attention to adequacy assessment and meticulous observation of samples are critical in order to reduce the discrepancy between cytologyhistological diagnoses. Though the percentage of correct diagnoses in EUS-FNAC results is relatively inferior compared to that from histological diagnosis, statistical results, such as diagnostic accuracy, were satisfactory in several studies including ours. Therefore, EUS-FNAC can be encouraged as a first-line pathologic examination for pancreatic lesion with high clinical suspicion of malignancy when patient safety and financial benefits are the priority.

\section{Conflicts of Interest}

No potential conflict of interest relevant to this article was reported.

\section{Acknowledgments}

This paper was supported by Seoul National University College of Medicine in 2014.

\section{REFERENCES}

1. Jung KW, Won YJ, Kong HJ, Oh CM, Lee DH, Lee JS. Cancer statistics in Korea: incidence, mortality, survival, and prevalence in 2011. Cancer Res Treat 2014; 46: 109-23.

2. Matsumoto K, Takeda Y, Harada K, Horie Y, Yashima K, Murawaki Y. Effect of pancreatic juice cytology and/or endoscopic ultrasoundguided fine-needle aspiration biopsy for pancreatic tumor. J Gastroenterol Hepatol 2014; 29: 223-7.

3. Bluen BE, Lachter J, Khamaysi I, et al. Accuracy and quality assessment of EUS-FNA: a single-center large cohort of biopsies. Diagn Ther Endosc 2012; 2012: 139563.

4. Hewitt MJ, McPhail MJ, Possamai L, Dhar A, Vlavianos P, Monahan KJ. EUS-guided FNA for diagnosis of solid pancreatic neoplasms: a meta-analysis. Gastrointest Endosc 2012; 75: 319-31.

5. Yoshinaga S, Suzuki H, Oda I, Saito Y. Role of endoscopic ultra- sound-guided fine needle aspiration (EUS-FNA) for diagnosis of solid pancreatic masses. Dig Endosc 2011; 23 Suppl 1: 29-33.

6. Kim TH, Choi KH, Song HS, Kim JW, Jeon BJ. Histology combined with cytology by endoscopic ultrasound-guided fine needle aspiration for the diagnosis of solid pancreatic mass and intra-abdominal lymphadenopathy. Gut Liver 2013; 7: 605-10.

7. Matsuyama M, Ishii H, Kuraoka K, et al. Ultrasound-guided vs endoscopic ultrasound-guided fine-needle aspiration for pancreatic cancer diagnosis. World J Gastroenterol 2013; 19: 2368-73.

8. Fisher L, Segarajasingam DS, Stewart C, Deboer WB, Yusoff IF. Endoscopic ultrasound guided fine needle aspiration of solid pancreatic lesions: performance and outcomes. J Gastroenterol Hepatol 2009; 24: 90-6.

9. Ardengh JC, Lopes CV, de Lima LF, et al. Diagnosis of pancreatic tumors by endoscopic ultrasound-guided fine-needle aspiration. World J Gastroenterol 2007; 13: 3112-6.

10. Varadarajulu S, Tamhane A, Eloubeidi MA. Yield of EUS-guided FNA of pancreatic masses in the presence or the absence of chronic pancreatitis. Gastrointest Endosc 2005; 62: 728-36.

11. Mitsuhashi T, Ghafari S, Chang CY, Gu M. Endoscopic ultrasoundguided fine needle aspiration of the pancreas: cytomorphological evaluation with emphasis on adequacy assessment, diagnostic criteria and contamination from the gastrointestinal tract. Cytopathology 2006; 17: 34-41.

12. Erickson RA, Sayage-Rabie L, Beissner RS. Factors predicting the number of EUS-guided fine-needle passes for diagnosis of pancreatic malignancies. Gastrointest Endosc 2000; 51: 184-90.

13. Kawamoto S, Siegelman SS, Hruban RH, Fishman EK. Lymphoplasmacytic sclerosing pancreatitis (autoimmune pancreatitis): evaluation with multidetector CT. Radiographics 2008; 28: 157-70.

14. Abraham SC, Leach S, Yeo CJ, et al. Eosinophilic pancreatitis and increased eosinophils in the pancreas. Am J Surg Pathol 2003; 27: $334-42$.

15. Moriya T, Kimura W, Hirai I, et al. Pancreatic schwannoma: Case report and an updated 30-year review of the literature yielding 47 cases. World J Gastroenterol 2012; 18: 1538-44.

16. Navina S, McGrath K, Chennat J, et al. Adequacy assessment of endoscopic ultrasound-guided, fine-needle aspirations of pancreatic masses for theranostic studies: optimization of current practices is warranted. Arch Pathol Lab Med 2014; 138: 923-8. 